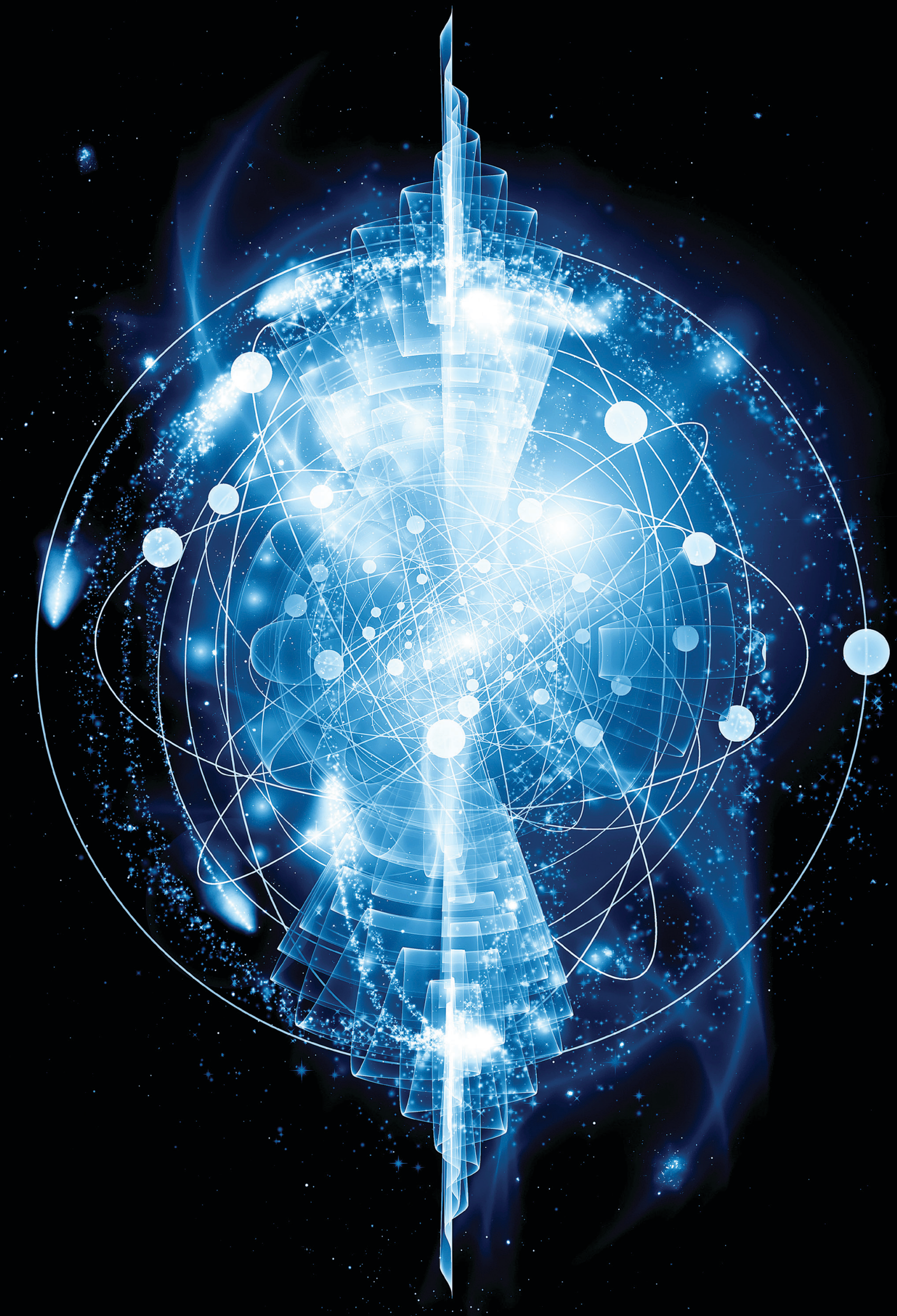




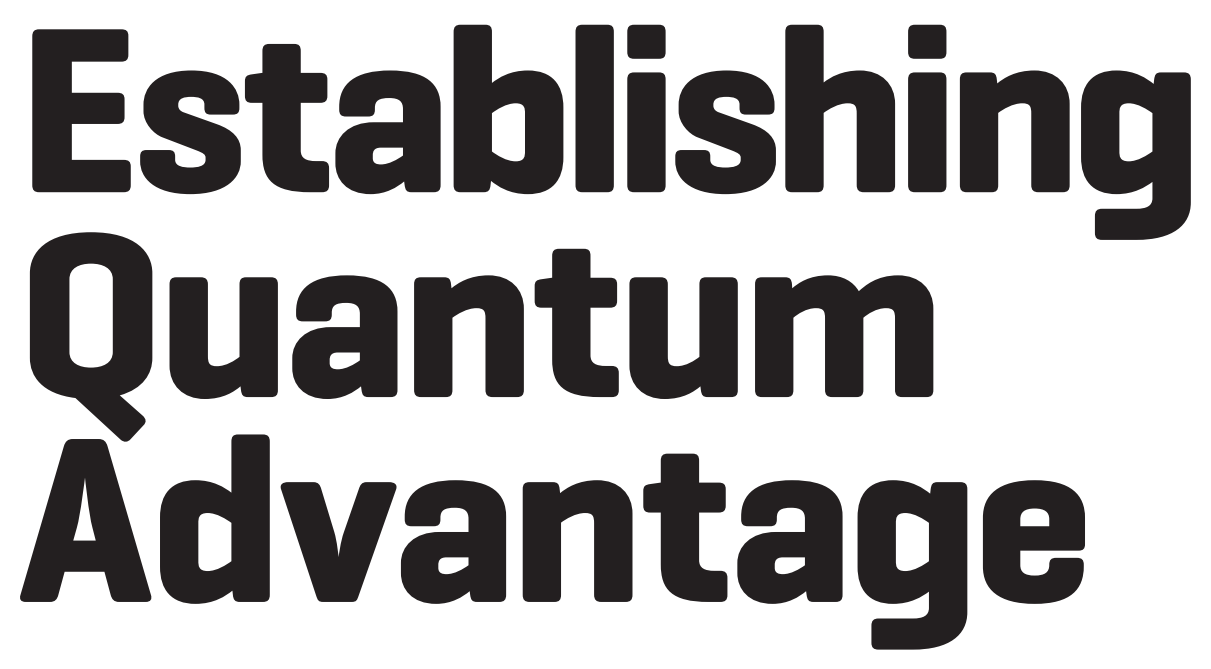

What are quantum computers good for? This essay
reviews the progress toward proving a quantum advantage
over classical computing.

ByAdam Bouland

DOI: $10.1145 / 2983543$

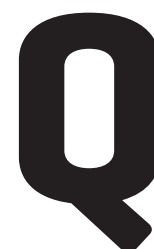

uantum computing is a method of manipulating tiny particles, such as individual photons or electrons, to process information. These particles obey the laws of quantum mechanics, which are fundamentally different from the laws of physics observed in day-to-day life. For example, particles can hold different positions and momenta simultaneously - a property known as quantum superposition. By harnessing the strange laws of quantum mechanics, quantum computing promises to provide an exponential advantage over classical computation. In other words, quantum computers could solve some problems in time, which scales polynomially in the input size, whereas a classical computer would require time that scales exponentially in the input

size. This "quantum leap" in computational power is widely proclaimed as revolutionary in popular media. In 2013 Google uploaded a video to YouTube of a scientist who claimed in addition to solving more mundane optimization problems, quantum computers might even help us determine whether or not extraterrestrials exist [1]. In the face of such speculative pronouncements, it is natural to ask exactly what problems quantum computers (once they're constructed) will be able to solve considerably faster than classical computers, such as laptops and smartphones. In other words, what are the "killer apps" of quantum computing? And is there evidence that proves classical computers (which we are far better at constructing) cannot perform these tasks efficiently? In this article we review the problems that show a quantum advantage, discuss the state of mathematically proving this advantage, and describe recent research on sampling tasks, which might provide the first experimental demonstration of quantum advantage in the near future.

\section{TASKS WITH QUANTUM ADVANTAGE}

There are a number of problems where quantum computing may potentially have an exponential advantage over classical computation. For example, quantum computers would have the incredible power to break most of the modern cryptography. In 1994, Peter Shor demonstrated quantum computers could factor an $n$-bit number in time that scales polynomially in $n$ [2]. In contrast, the best-known classical algorithm takes time that scales exponentially in $n$. Many believe more efficient algorithms do not exist, and this belief is the basis of much of modern cryptography. In fact, quantum computers could efficiently break a number of public-key cryptosystems, including RSA and Diffie-Hellman, which are used in electronic commerce and banking. So an adversary with a quan- 


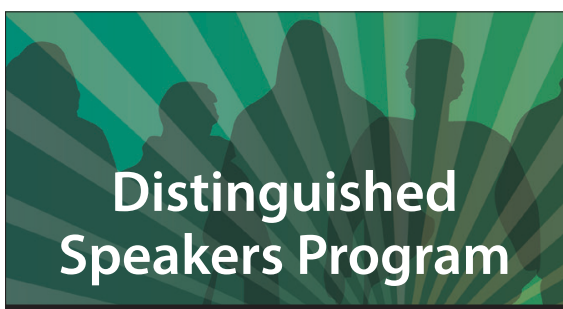

http://dsp.acm.org

\section{Students and faculty} can take advantage of ACM's Distinguished Speakers Program to invite renowned thought leaders in academia, industry and government to deliver compelling and insightful talks on the most important topics in computing and IT today. ACM covers the cost of transportation for the speaker to travel to your event. tum computer could break into customers' bank accounts, read encrypted emails, and interfere with online shopping. Fortunately, there are some alternative cryptographic protocols, such as lattice-based cryptography, which may be immune to quantum attacks. Nevertheless, the development of quantum computers will radically change the nature of cryptography.

Although the cryptographic applications of quantum computers are well advertised in the popular press, a potential killer app of quantum computing, and one that is rarely mentioned, is the task of simulating quantum mechanics. This was part of the original motivation for building quantum computers. In 1982, Feynman observed to simulate a quantum system of $n$ particles, one needed to keep track of roughly $2^{n}$ parameters. Therefore, he reasoned it might be difficult to simulate large quantum systems on classical computers. This has turned out to be true in practice as simulating complex quantum mechanical processes, such as protein folding, are extremely difficult. In practice, researchers use various simplifications or approximations to quantum mechanics, such as density functional theory, to estimate their quantities of interest. However, these heuristic approaches work in limited cases. If quantum computers are built, this will allow us to simulate many quantum processes to high precision. These simulations would have many applications in pharmaceuticals, materials science, and quantum chemistry.

Additionally, we believe quantum computers can provide an exponential advantage for more obscure problems, like computing certain topological invariants of knots. Strangely these problems have been shown to be complete for quantum computing. This means that any quantum computation can be recast as a problem of computing topological knot invariants. However, it is unclear what the application of such results would be outside of knot theory.

Interestingly, we do not have evidence that quantum computers will have an advantage solving $N P$-hard problems. An $N P$-hard problem is a problem for which one can efficiently verify the solution, but cannot necessarily find the answer efficiently due to the fact that there are exponentially many possible solutions. For example, finding the most efficient route to visit $n$ cities is an $N P$-complete problem. There are $n$ factorial orderings of cities one could visit, and it is difficult to find what ordering results in the shortest travel time. Such problems commonly arise in optimization, where it is extremely difficult to find the best solution to a complex optimization problem. Although D-Wave, a commercial quantum computing company, is working to construct quantum computers to solve such optimization problems, it is unclear if quantum computers will have an advantage at this task. There is evidence that quantum computers cannot efficiently solve $N P$-hard optimization problems on worst-case inputs. In particular, we know quantum computers cannot efficiently brute-force search over an exponentially large set of all possible answers to find a solution. In other words, quantum computers aren't any better at finding "a needle in a haystack" than a normal computer, up to polynomial factors. Therefore, it is widely expected that quantum computers will not be able to solve $N P$-hard problems exponentially faster than classical computers on worst-case instances. It remains open whether quantum computers can provide an advantage on natural or average-case inputs of $N P$-hard problems, as they are often much easier than worst-case inputs. This is also the subject of a current experimental investigation by $\mathrm{D}$-Wave and the research community.

\section{LOCKING IN THE ADVANTAGE}

So far we know quantum computers can solve factoring, simulate quantum mechanics, and compute knot invariants exponentially faster than the bestknown classical algorithm. But how do we know these problems can't be solved with your laptop? What if a faster classical algorithm exists, but algorithms designers simply haven't thought of it yet? Can we rule out this possibility?

Unfortunately, the short answer to this question is "no." Ruling out the existence of efficient classical algorithms for such problems is an extremely difficult mathematical problem. In essence, this is difficult because infinitely many possible algorithms exist, essentially 
one for each computer program one could write. While it is obvious most computer programs one could write will not solve factoring, it is very difficult to argue none of those algorithms would work, or none of those that work would be efficient. It is impossible to go through them individually and figure out why they do not work, as doing so would take an infinitely long time.

In fact, if it can be proven that quantum computers have an exponential advantage for any of these tasks, it would answer a major open problem in computational complexity theory. An established result is a classical computer using polynomial memory and exponential time can also solve any problem that can be solved by a quantum computer. Such an algorithm is called a PSPACE (polynomial space) algorithm, because it uses a reasonable amount of memory but an unreasonably long amount of processing time. Therefore, to prove quantum computers have an exponential advantage over classical computers, it would require proving a more efficient algorithm with a faster runtime could not solve any PSPACE problem. This is known as the $P$ vs. PSPACE problem. Interestingly, this is an open problem, which is closely related to the $P$ vs. $N P$ problem. The latter problem carries a \$1M prize from the Clay Mathematics Institute for its solution.

Mathematicians have a long way to go before they can solve either of these problems. In particular, we know several proof techniques, which are useful in other areas of complexity theory, will not be able to resolve the $P$ vs. $N P$ problem, the $P$ vs. PSPACE problem, or the problem of proving a quantum advantage. For instance, imagine a quantum computer or a classical computer is given access to a subroutine, which computes some arbitrary function $f$ in constant time. Each computer is provided access to this function, as an API, but has no control over how it is being solved in the back end. The strange thing is, there exist some functions $f$ that render quantum computers and classical computers equally powerful in this setup. So any argument made that is independent of the APIs available cannot prove quantum computers are more powerful than classical computers. This is known as the "relativ-

\section{By harnessing the strange laws of quantum mechanics, quantum computing promises to provide an advantage over classical computation.}

ization" barrier in complexity theory. Unfortunately, most arguments we know to prove separations in power are invariant under giving each computer access to the same APIs, so any proof of that form will not be able to resolve this problem.

Since common proof techniques do not work for this problem, mathematicians do not know where to begin to approach these problems. We do know how to prove some similar, but much weaker, statements. For example, we can sometimes show algorithms with power $A$ cannot be simulated by algorithms with power $B$, but only when the power of $A$ is drastically more powerful than $B$. Even these results are considered breakthroughs in the field. As a result, it seems there is no hope of definitively ruling out the possibility of classical algorithms for our applications of quantum computing - at least in the near future. Furthermore, it is not known solving the above problems in classical polynomial time would have any unexpected consequences in structural complexity theory, so we cannot base our belief in quantum advantage for these problems on other assumptions in complexity theory. It appears our quest to mathematically prove quantum advantage is at a dead end.

\section{POTENTIAL NEXT STEPS}

In the face of these obstacles, there are several approaches one can take toward establishing a quantum advantage. One approach is to dismiss the concerns of the previous section as merely theoretical. Rather than mathematically proving quantum advantage for these problems, one can simply try to build a quantum computer to solve very large instances of these hard problems. The hope is one will empirically, rather than theoretically, demonstrate a quantum advantage. For example, if an integer is factored with a quantum computer, which is larger than what any classical computer has ever factored (e.g. the RSA 2048-digit challenge key), this would be an impressive experimental evidence of quantum advantage.

While this is a long-term goal of the experimental quantum computing community, we cannot yet build quantum computers that can factor large numbers, despite much experimental progress. So far we can only build prototype quantum devices, and the largest number ever factored by these prototypes using Shor's algorithm is currently 21. So it seems an empirical demonstration of quantum advantage by factoring large integers is beyond our present experimental abilities. A natural practical question arises: What are these prototype quantum computers good for? Can we find some problems that can be solved with a small quantum computer, which still give an advantage over classical computation? Perhaps quantum advantage could then be empirically tested using our prototype quantum devices, before we are able to build bigger and better quantum computers capable of factoring.

Another, seemingly opposite approach is to dig deeper into the realm of theory, and find different problems, which quantum computers have an advantage solving. The hope is that one may be able to base one's belief of quantum supremacy on different complexity-theoretic assumptions other than the hardness of factoring or simulating quantum mechanics. Perhaps using this approach, one could provide new theoretical evidence for quantum advantage.

\section{A NEW APPROACH: SHOWING QUANTUM ADVANTAGE FOR SAMPLING}

Amazingly the two approaches mentioned have recently converged. It turns out by studying a different type of computational task, known as a sampling problem, one can establish quantum advantage on different theo- 
retical grounds. Furthermore, these sampling problems might be easier to implement practically than factoring, and might lend themselves to experimental implementation before we have better quantum devices that can factor. In short, one might be able to provide both stronger experimental evidence and further theoretical evidence of a quantum advantage.

Sampling problems are entirely different from how we normally think of computation. Instead of asking for a particular output for every input, a sampling task asks you to sample from a particular probability distribution on outputs for every input. For example, a sampling task might be: Given a number $x$ in binary, sample a random binary string that contains $x$ as a substring. The basic idea is to consider what kind of probability distributions (rather than deterministic functions) can be sampled from with quantum computers. This is an entirely different notion of a computational task.

Recently, research has shown quantum computers can perform sampling tasks that classical computers cannot do. One can show these are impossible for classical computers, assuming a widely held conjecture in complexity theory known as the "non-collapse of the polynomial hierarchy" (a generalization of the belief that $P \neq N P$ ). As a bonus, often these sampling tasks can be performed with weaker quantum computers that do not necessarily have the ability to factor, so they are particularly well suited to be performed by the prototype quantum computers we are able to build experimentally thus far. For example, the boson-sampling model of Aaronson and Arkhipov [3] can be implemented using linear optical quantum computers without interactions between the photons. In contrast, factoring with optical quantum computers requires nonlinear interactions between the photons, which are difficult to implement. Similarly, the "Temporally Unstructured Quantum Computing" model of Bremner, Jozsa, and Shepherd [4] can be implemented using operations that commute with one another. These may be easier to implement in practice on superconducting qubit architectures. Both these models can perform sampling tasks that are difficult for classi-

\section{It is widely expected quantum computers will not be able to solve $N P$-hard problems exponentially faster than classical computers.}

cal computers, and yet may be easier to implement than factoring. Even performing these tasks on small prototype quantum computers, with around 20-40 quantum bits, would be beyond the capabilities of a laptop. In contrast, factoring 40-bit numbers can be performed on a laptop.

Admittedly, these sampling tasks are less useful than factoring or simulating quantum mechanics. They sample from probability distributions, which do not help to solve any hard problems. However, their principal utility lies in demonstrating quantum advantage from prototype quantum computers, which will be constructed in the near future. Whatever these tasks are, they capture something that cannot be done with a classical computer.

There are some theoretical difficulties with this approach as well. To achieve the hardness of simulation results mentioned earlier, one has to consider the probability distributions output by perfect, noiseless quantum devices. But in practice, quantum computers are inherently noisy, due to the presence of unwanted interactions between the quantum computer and its environment. By using clever quantum error correcting codes, one can decrease the amount of noise such that it doesn't affect the computation much. This residual noise is irrelevant for solving problems like factoring, but is much more troublesome for these sampling tasks. In fact, it is troublesome enough to foil the hardness results for the sampling tasks mentioned previously. To overcome this obstacle, a number of researchers have sought to prove hardness of simulation under more realistic error models.
So far researchers can only prove such results under unproven mathematical conjectures. It seems very difficult to remove these sorts of assumptions from the proofs. But if one proves the conjectures, it would show that even noisy quantum devices have supremacy over classical devices for sampling tasks (assuming the widely-held generalization of $P \neq N P$ ).

\section{CONCLUSION}

Quantum computers are capable of performing a number of tasks exponentially faster than classical computation devices. In the long term, we expect to find a quantum advantage for factoring and simulating quantum systems, which will fundamentally change cryptography, biotechnology, quantum chemistry and materials science. However, mathematically proving quantum advantage is surprisingly difficult, and empirically demonstrating a quantum advantage for factoring is beyond our current experimental capabilities. In the near future, we may have access to prototype quantum computers acting on a few tens of quantum bits. Rather than using these computers to factor integers, it is likely we will use them first to perform sampling tasks that show a quantum advantage over classical devices. These sampling tasks will be a stepping-stone on our way to unlocking the full power of quantum computation.

\section{References}

[1] Google and NASA's Quantum Artificial Intelligence Lab. YouTube. October 11, 2013

https://www.youtube.com/ watch?v=CMdHDHEuOUE, Time 4:57

[2] Shor, P. W. Algorithms for quantum computation discrete logarithms and factoring Proc. IEEE FOCS'94, [1994],124-134.

[3] Aaronson, S., and Arkhipov, A. The computational complexity of linear optics. Theory of Computing 9, 4 [2013], 143-252.

[4] Bremner, M. J., Jozsa, R., and Shepherd, D.J. Classical simulation of commuting quantum computations implies collapse of the polynomial hierarchy. In Proceedings of the Royal Society of London A: Mathematical, Physical and Engineering Sciences 467, [2010], 459.

\section{Biography}

Adam Bouland is a Ph.D. student at MIT studying theoretical computer science. Prior to coming to MIT, he completed his undergraduate at Yale and his master's at the University of Cambridge under a Marshall Scholarship. His research focuses on classifying the computational power of weak models of quantum computing as well as finding connections between quantum computing theory and physics.

[ 2016 Copyright held by Owner[s]/Author[s]. Publication rights licensed to ACM. $1528-4972 / 16 / 09 \$ 15.00$ 